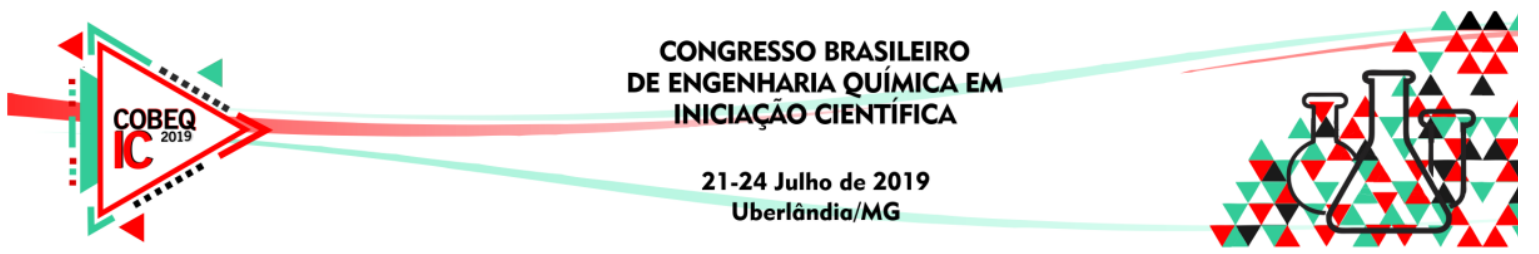

\title{
ESTUDO DOS COEFICIENTES DE TRANSFERÊNCIA DE CALOR EM UM PASTEURIZADOR COM TROCADORES TIPO SERPENTINA EM CASCO
}

\author{
F. G. DITCHUN ${ }^{1}$, G. RUSSO ${ }^{2}$ e J. A. W. GUT ${ }^{2,3}$ \\ ${ }^{1}$ Departamento de Engenharia de Alimentos, Faculdade de Tecnologia Termomecanica \\ ${ }^{2}$ Departamento de Engenharia Química, Universidade de São Paulo \\ ${ }^{3}$ Food Research Center (FoRC), Universidade de São Paulo \\ E-mail para contato: fer.nanda_ditchun@hotmail.com
}

\begin{abstract}
RESUMO - A pasteurização é um tratamento térmico brando, aplicado a alimentos líquidos por meio de trocadores de calor, que é dividido em três etapas: aquecimento, retenção e resfriamento. $\mathrm{O}$ aquecimento em fluxo contínuo por microondas focalizadas é uma tecnologia que vem sendo estudada por ter potencial para substituir trocadores de calor para promover um aquecimento mais rápido, reduzindo a perda de atributos de qualidade. Este trabalho estuda os coeficientes de transferência de calor envolvidos no processamento térmico assistido por microondas em uma unidade piloto Microwave Lab25-UHT/HTST EHVH nas etapas de pré-aquecimento, retenção e resfriamento. Diferentes condições de processo foram contempladas para a água: vazão do produto $(0,4$ a $2,0 \mathrm{~L} / \mathrm{min})$ e temperatura de processo $\left(60\right.$ a $\left.120{ }^{\circ} \mathrm{C}\right)$. Os coeficientes de transferência de calor foram obtidos a partir dos dados experimentais no pré-aquecedor, no resfriador e no tubo de retenção (perda de calor para o ambiente). Este trabalho contribuirá com o desenvolvimento de um modelo de simulação de processo para determinação da distribuição de temperatura e tempo de residência do produto, tendo em vista a avaliação dos efeitos da alta temperatura sobre atributos de segurança e qualidade.
\end{abstract}

\section{INTRODUÇÃO}

A pasteurização assistida por micro-ondas é uma tecnologia que vem sendo estudada por ter potencial para substituir trocadores de calor no tratamento térmico de produtos líquidos, por promover a redução do tempo de exposição à alta temperatura no aquecimento, maior eficiência energética e melhora na qualidade do alimento (Chandrasekaran et al., 2013), reduzindo a perda de atributos de qualidade e o sobre-processamento, ao mesmo tempo que atinge os níveis de letalidade desejáveis.

\section{OBJETIVO}

O objetivo deste trabalho é estudar os coeficientes de transferência de calor nas etapas de pré-aquecimento, retenção e resfriamento em um processo de pasteurização assistido por microondas.

\section{MÉTODOS E PROCEDIMENTOS}






Para os ensaios experimentais foi usada a unidade piloto Microwave Lab25-UHT/HTST EHVH (MicroThermics, EUA) com seções de pré-aquecimento, aquecimento por micro-ondas, tubo de retenção e resfriamento. Como o objetivo do estudo são os trocadores de calor tipo serpentina em casco e o tubo de retenção, o aquecedor por micro-ondas não foi utilizado nos ensaios.

Figura 1 - Pasteurizador Microwave Lab25-UHT/HTST EHVH (MicroThermics, EUA).

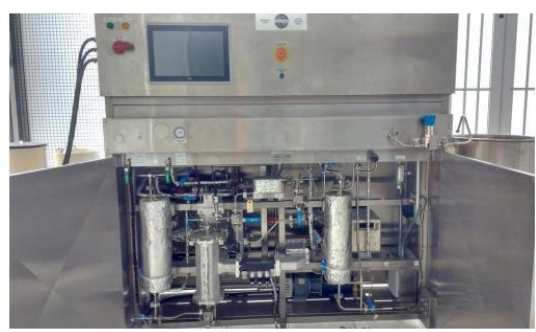

Foi processada água, variando a vazão do produto $(0,4$ a $2,0 \mathrm{~L} / \mathrm{min})$ e temperatura de processo $\left(60\right.$ a $\left.120{ }^{\circ} \mathrm{C}\right)$. Termopares registraram as temperaturas de entrada e saída nos trocadores. Propriedades termofísicas da água foram calculadas para as faixas de temperatura estudadas.

Os calores nos lados quente e frio foram calculados pelas Equações 1 e 2, respectivamente. Os subscritos $q$ (quente) e $f$ (frio) indicam os lados do trocador e os subscritos $e$ e $s$ representam entrada e saída.

$$
\begin{aligned}
& q=\dot{m}_{q} \cdot C_{p, q} \cdot\left(T_{q, e}-T_{q, s}\right) \\
& q=\dot{m}_{f} \cdot C_{p, f} \cdot\left(T_{f, e}-T_{f, s}\right)
\end{aligned}
$$

em que $q$ é a taxa de transferência de calor (W), m é a vazão mássica do fluido $(\mathrm{kg} / \mathrm{s}), C_{p}$ é o calor específico médio do fluido $(\mathrm{J} / \mathrm{kg} . \mathrm{K})$ e T é a temperatura do fluido $\left({ }^{\circ} \mathrm{C}\right)$. A perda de calor no tubo de retenção corresponde ao balanço de energia para o lado quente de um trocador de calor (Equação 1), considerando o ar ambiente como o lado frio isotérmico.

O coeficiente global de transferência de calor foi calculado pela Equação 3.

$$
q=U \cdot A \cdot \Delta \bar{T}_{l n}
$$

em que $q$ é a taxa de transferência de calor (W), $U$ é o coeficiente global de transferência de calor $\left(\mathrm{W} / \mathrm{m}^{2} . \mathrm{K}\right), A$ é a área de referência para transferência de calor $\left(\mathrm{m}^{2}\right)$ e $\Delta \bar{T}_{l n}$ é a média logarítmica da diferença de temperatura $\left({ }^{\circ} \mathrm{C}\right)$.

O coeficiente global de transferência de um trocador de calor tubular pode ser representado pela Equação 4, levando em consideração as resistências térmicas condutivas e convectivas associadas em série.

$$
\frac{1}{U \cdot A_{\text {ref }}}=\frac{1}{h_{e} \cdot A_{e}}+\frac{e}{k \cdot A_{l m}}+\frac{1}{h_{i} \cdot A_{i}}
$$



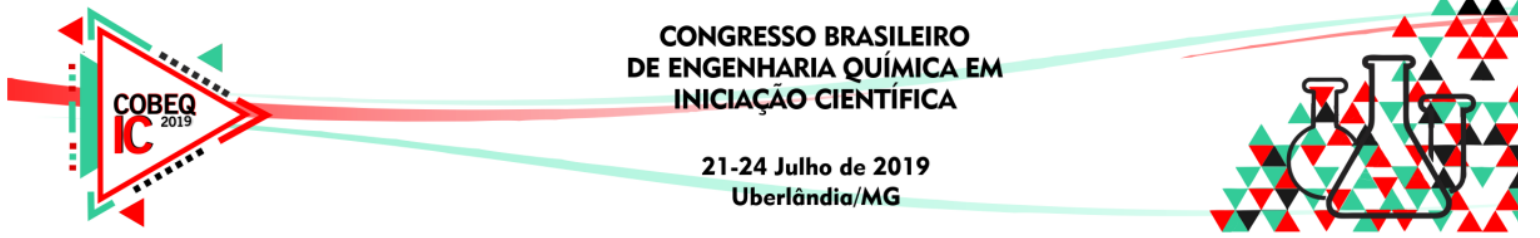

em que $h_{i}$ é o coeficiente convectivo do fluido interno ao tubo $\left(\mathrm{W} / \mathrm{m}^{2} . \mathrm{K}\right), h_{e}$ é o coeficiente convectivo do fluido externo ao tubo $\left(\mathrm{W} / \mathrm{m}^{2} . \mathrm{K}\right), A_{e}$ é a área para troca externa do tubo $\left(\mathrm{m}^{2}\right), A_{i}$ é a área para troca interna do tubo $\left(\mathrm{m}^{2}\right), A_{r e f}$ é a área de referência $\left(\mathrm{m}^{2}\right), e$ é a espessura do tubo $(\mathrm{m}), k$ é a condutividade térmica do material do tubo (W/m.K), e $A_{l m}$ é a área logarítmica média do tubo $(\mathrm{m})$. A área de referência adotada foi a área externa da serpentina em contato com o fluido de utilidade.

O número de Reynolds foi calculado pela Equação 5.

$$
R e=\frac{d_{i} \cdot v \cdot \rho}{\mu}
$$

em que $R e$ é o número de Reynolds (adimensional), $d_{i}$ é o diâmetro interno do tubo $(\mathrm{m}), v$ é a velocidade média de escoamento do fluido $(\mathrm{m} / \mathrm{s}), \rho$ é a densidade do fluido $\left(\mathrm{kg} / \mathrm{m}^{3}\right)$ e $\mu$ é a viscosidade do fluido newtoniano (Pa.s).

E o número de Prandtl foi calculado pela Equação 6.

$$
\operatorname{Pr}=\frac{\mu \cdot C_{p}}{k}
$$

em que $\operatorname{Pr}$ é o número de Prandtl (adimensional), $\mu$ é a viscosidade do fluido newtoniano (Pa.s), $C_{p}$ é o calor específico médio do fluido $(\mathrm{J} / \mathrm{kg} . \mathrm{K})$ e $k$ é a condutividade térmica do material do tubo (W/m.K).

Foram propostos seis modelos matemáticos que correlacionam o coeficiente global de troca térmica $(U)$ com Reynolds $(R e)$ e Prandtl $(P r)$, conforme a Tabela 1. Sendo o Modelo F proposto por Coronel et al. (2008), a correlação foi apresentada entre $U$ e $R e$ para um trocador de calor helicoidal similar ao estudado neste trabalho. $\mathrm{O}$ formato destas correlações deriva das resistências térmicas que compõem o $U$ de um trocador de calor. Os subscritos $p$ e $u$ representam produto e utilidade, respectivamente.

Tabela 1 - Equações propostas que correlacionam o $U$ com $R e$ e $P r$.

\begin{tabular}{|c|c|}
\hline Modelo & Equação proposta \\
\hline $\mathrm{A}$ & $\frac{1}{U \cdot A}=a+\frac{b}{R e_{p}^{c} \cdot P r_{p}^{f}}+\frac{d}{R e_{u}^{e} \cdot P r_{u}^{g}}$ \\
\hline $\mathrm{B}$ & $\frac{1}{U \cdot A}=a+\frac{b}{R e_{p}^{c} \cdot P r_{p}^{1 / 3}}+\frac{d}{R e_{u}^{e} \cdot P r_{u}^{1 / 3}}$ \\
\hline $\mathrm{C}$ & $\frac{1}{U \cdot A}=a+\frac{b}{R e_{p}^{c}}+\frac{d}{R e_{u}^{e}}$ \\
\hline $\mathrm{D}$ & $\frac{1}{U \cdot A}=a+\frac{b}{R e_{p}^{c} \cdot P r_{p}^{f}}$ \\
\hline $\mathrm{E}$ & $\frac{1}{U \cdot A}=a+\frac{b}{R e_{p}^{c} \cdot P r_{p}^{1 / 3}}$ \\
\hline $\mathrm{F}$ & $\frac{1}{U \cdot A}=a+\frac{b}{R e_{p}^{c}}$ \\
\hline
\end{tabular}




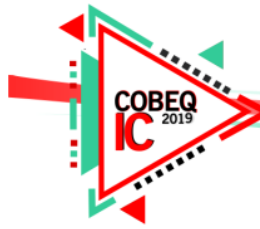

\section{RESULTADOS E DISCUSSÃO}

As Tabelas 2 a 4 mostram os parâmetros ajustáveis dos modelos propostos para as seções de aquecimento, resfriamento e retenção, obtidos a partir da minimização do erro quadrático no cálculo de $U$ pela ferramenta Solver do programa MS Excel.

A Tabela 2 apresenta os parâmetros ajustáveis dos modelos matemáticos propostos para a seção de aquecimento. Para o pré-aquecedor, o número de Reynolds do produto variou de $1,5.10^{3}$ a $1,1.10^{4}$ e o número de Prandtl do produto variou de 3,1 a 5,4. Nos modelos A, B e C, o número de Reynolds da utilidade variou de $4,9.10^{3}$ a $1,0.10^{4}$ e o número de Prandtl da utilidade variou de 1,5 a 3,0 .

Tabela 2 - Parâmetros ajustáveis dos modelos matemáticos propostos da seção de aquecimento.

\begin{tabular}{|c|c|c|c|c|c|c|c|c|c|}
\hline Modelo & $a$ & $b$ & $c$ & $d$ & $e$ & $f$ & $g$ & $\sum$ Erro $^{2}$ & $R^{2}$ \\
\hline A & $6,76 \cdot 10^{-5}$ & 12,4 & 0,925 & 5,08 & 1,00 & 0,667 & 0,285 & 0,0792 & 0,975 \\
\hline B & $1,83 \cdot 10^{-4}$ & 4,58 & 0,865 & 0,510 & 0,766 & & & 0,133 & 0,967 \\
\hline C & $5,41 \cdot 10^{-4}$ & 1,72 & 0,800 & 0,00218 & 0,800 & & & 0,202 & 0,950 \\
\hline D & $1,53 \cdot 10^{-4}$ & 3,47 & 0,799 & & & 0,336 & & 0,244 & 0,932 \\
\hline E & $1,48 \cdot 10^{-4}$ & 3,44 & 0,800 & & & & & 0,247 & 0,929 \\
\hline F & $2,78 \cdot 10^{-4}$ & 2,04 & 0,800 & & & & & 0,393 & 0,913 \\
\hline
\end{tabular}

A Tabela 3 apresenta os parâmetros ajustáveis dos modelos matemáticos propostos para a seção de resfriamento. Para o resfriador, o número de Reynolds do produto variou de $9,1.10^{2}$ a $1,0.10^{4}$ e o número de Prandtl do produto variou de 2,7 a 6,6. Nos modelos A, B e C, o número de Reynolds da utilidade variou de $9,3.10^{2}$ a $1,9.10^{3}$ e o número de Prandtl da utilidade variou de 5,2 a 11,9 .

Tabela 3 - Parâmetros ajustáveis dos modelos matemáticos propostos da seção de resfriamento.

\begin{tabular}{|c|c|c|c|c|c|c|c|c|c|}
\hline Modelo & $a$ & $b$ & $c$ & $d$ & $e$ & $f$ & $g$ & $\sum$ Erro $^{2}$ & $R^{2}$ \\
\hline $\mathrm{A}$ & $8,42 \cdot 10^{-5}$ & 2,34 & 0,849 & 2,77 & 0,870 & 0,161 & 0,140 & 0,0218 & 0,990 \\
\hline $\mathrm{B}$ & $3,35 \cdot 10^{-5}$ & 3,21 & 0,820 & 2,82 & 0,835 & & & 0,0408 & 0,980 \\
\hline $\mathrm{C}$ & $6,78 \cdot 10^{-4}$ & 1,26 & 0,800 & 1,05 & 0,800 & & & 0,0203 & 0,991 \\
\hline $\mathrm{D}$ & $2,15 \cdot 10^{-3}$ & 1,23 & 0,644 & & & 0,325 & & 0,130 & 0,914 \\
\hline $\mathrm{E}$ & $2,17 \cdot 10^{-3}$ & 1,27 & 0,648 & & & & & 0,131 & 0,912 \\
\hline $\mathrm{F}$ & $2,24 \cdot 10^{-3}$ & 0,378 & 0,562 & & & & & 0,0726 & 0,954 \\
\hline
\end{tabular}

A Tabela 4 apresenta os parâmetros ajustáveis dos modelos matemáticos propostos para a seção de retenção. Para o tubo de retenção, o número de Reynolds do produto variou de 2,2.10 a $2,1 \cdot 10^{4}$ e o número de Prandtl do produto variou de 1,4 a 3,0. 

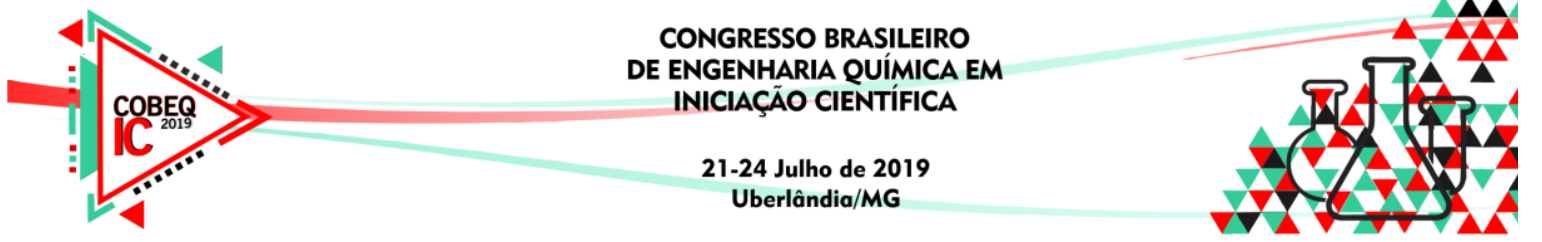

Tabela 4 - Parâmetros ajustáveis dos modelos matemáticos propostos da seção de retenção.

\begin{tabular}{|c|c|c|c|c|c|c|c|c|c|}
\hline Modelo & $a$ & $b$ & $c$ & $d$ & $e$ & $f$ & $g$ & $\sum$ Erro $^{2}$ & $R^{2}$ \\
\hline $\mathrm{D}$ & 0,714 & 103 & 0,682 & & & 0,221 & & 0,150 & 0,473 \\
\hline $\mathrm{E}$ & 0,758 & 388 & 0,852 & & & & & 0,157 & 0,452 \\
\hline $\mathrm{F}$ & 0,758 & 219 & 0,809 & & & & & 0,137 & 0,521 \\
\hline
\end{tabular}

Os Modelos A, B e C resultaram em melhores ajustes do que os Modelos D, E e F. Esses consideraram a influência dos fluidos de utilidade para a estimação do $U$ nas seções de troca térmica e estes a vazão dos fluidos de utilidade constante e que $U$ depende principalmente da vazão e propriedades do produto.

Os Modelos C e F consideram Pr constante. Os Modelos A, B, D e E consideram Pr para avaliar a influência da temperatura de processo, sendo nos Modelos B e E o expoente de $\mathrm{Pr}$ fixo no valor de 1/3, baseado na equação de Colburn (Bergman et al., 2015) e nos Modelos A e D estes parâmetros são estimados pela correlação de $U$. Nas seções de troca térmica, os parâmetros $f$ e $g$ do Modelo A distanciaram do valor proposto pela equação de Colburn, enquanto o Modelo D obteve resultados próximos à 1/3, expoente de $\operatorname{Pr}$ proposto para o Modelo E.

A Figura 2 mostra os gráficos referentes aos ajustes dos Modelos D, E e F para as seções de aquecimento, resfriamento e retenção.

Figura 2 - Ajuste dos Modelos D, E e F para as seções de aquecimento, resfriamento e retenção.

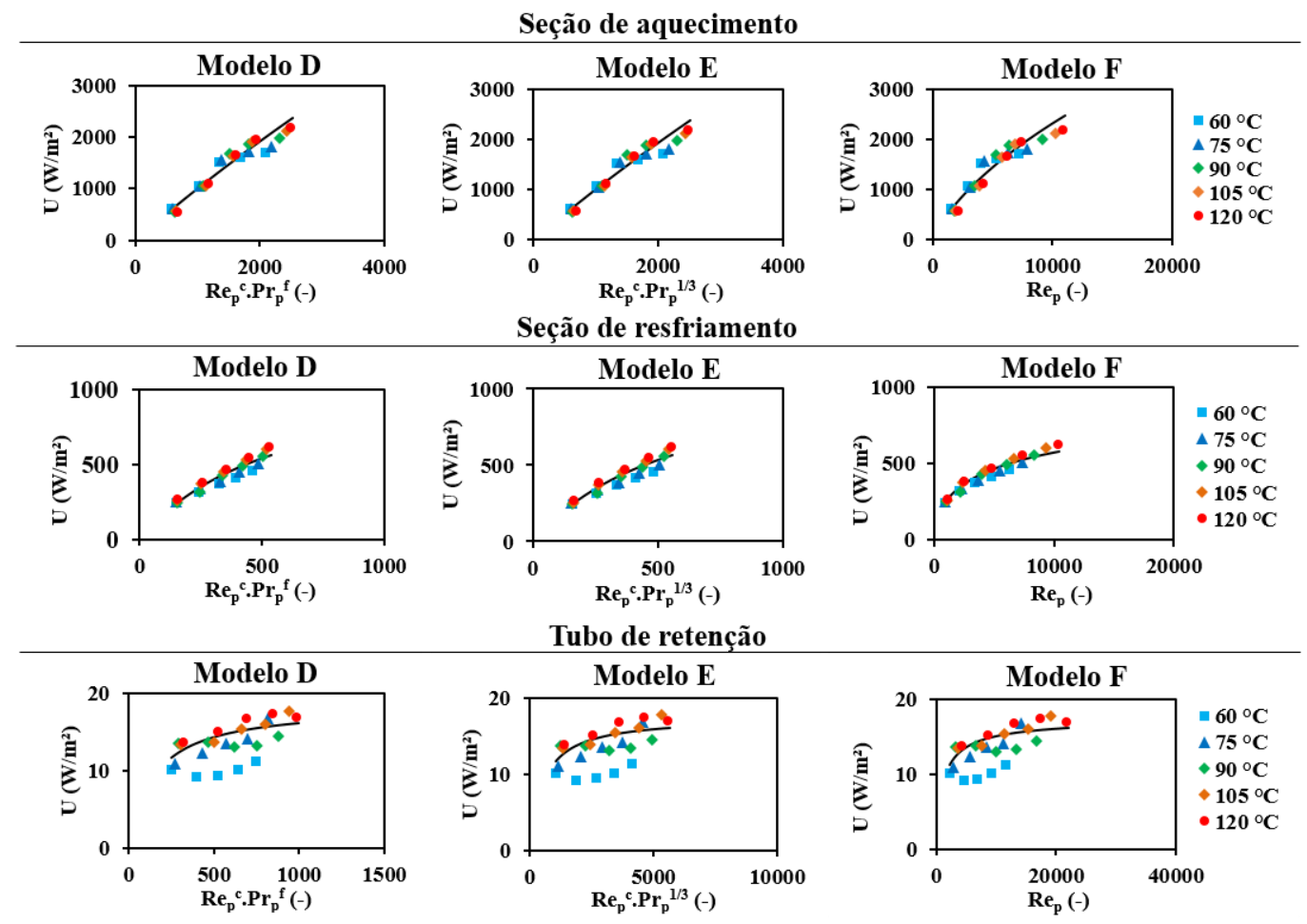




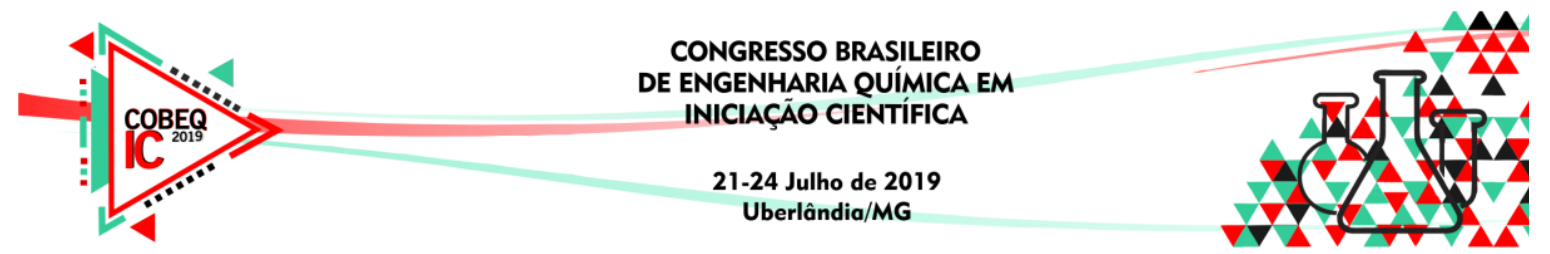

\section{CONCLUSÃO}

O melhor ajuste para o pré-aquecedor foi o Modelo A e para o resfriador foram os Modelos A e C. Comparado com as seções de troca térmica, a seção de retenção apresentou coeficientes de correlação insatisfatórios. O parâmetro $a$ influencia na altura da curva e os parâmetros $b$ e $c$ na curvatura dos modelos propostos.

Valores de $U$ são próximos para vazões baixas e mais afastados quanto maior a temperatura para vazões mais elevadas. Para uma mesma temperatura, os pontos se alinham ao variar a vazão, como se para cada temperatura houvesse um perfil de $U$. O aumento da temperatura para uma mesma vazão aumenta o $R e$, pois diminui a viscosidade do produto. Resultados com outros fluidos permitirão verificar a influência da reologia na troca térmica.

Ensaios devem ser realizados para determinação da taxa de transferência de energia no aquecedor por micro-ondas para que o processo possa ser modelado por completo.

\section{REFERÊNCIAS}

BERGMAN, T. et al. Fundamentos de transferência de calor e de massa. Tradução. 7. ed. Rio de Janeiro: Grupo Gen - LTC, 2015.

CHANDRASEKARAN, S.; RAMANATHAN, S.; BASAK, T. Microwave food processing-a review. Food Res. Int., v. 52, n. 1, p. 243-261, 2013.

CORONEL, P.; SIMUNOVIC, J.; SANDEEP, K. P.; CARTWRIGHT, G.D.; KUMAR, P. Sterilization solutions for aseptic processing using a continuous flow microwave system. J. Food Eng., v. 85, p. 528-536, 2008.

\section{AGRADECIMENTOS}

Fundação de Amparo à Pesquisa do Estado de São Paulo (FAPESP), processos 2018/08178-7 e 2013/07914-8. 